\title{
Front Matter: Volume 11279
}

, "Front Matter: Volume 11279," Proc. SPIE 11279, Terahertz, RF, Millimeter, and Submillimeter-Wave Technology and Applications XIII, 1127901 (8 April 2020); doi: $10.1117 / 12.2567562$

SPIE. Event: SPIE OPTO, 2020, San Francisco, California, United States 


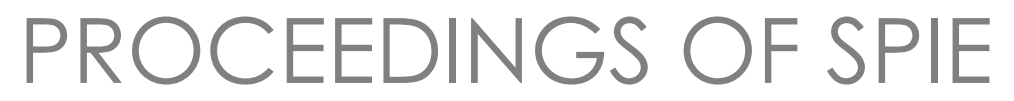

\section{Terahertz, RF, Millimeter, and Submillimeter-Wave Technology and Applications XIII}

Laurence P. Sadwick

Tianxin Yang

Editors

3-6 February 2020

San Francisco, California, United States

Sponsored and Published by

SPIE

Volume 11279 
The papers in this volume were part of the technical conference cited on the cover and title page. Papers were selected and subject to review by the editors and conference program committee. Some conference presentations may not be available for publication. Additional papers and presentation recordings may be available online in the SPIE Digital Library at SPIEDigitalLibrary.org.

The papers reflect the work and thoughts of the authors and are published herein as submitted. The publisher is not responsible for the validity of the information or for any outcomes resulting from reliance thereon.

Please use the following format to cite material from these proceedings:

Author(s), "Title of Paper," in Terahertz, RF, Millimeter, and Submillimeter-Wave Technology and Applications XIII, edited by Laurence P. Sadwick, Tianxin Yang, Proceedings of SPIE Vol. 11279 (SPIE, Bellingham, WA, 2020) Seven-digit Article CID Number.

ISSN: 0277-786X

ISSN: 1996-756X (electronic)

ISBN: 9781510633216

ISBN: 9781510633223 (electronic)

Published by

SPIE

P.O. Box 10, Bellingham, Washington 98227-0010 USA

Telephone +1 3606763290 (Pacific Time) · Fax +1 3606471445

SPIE.org

Copyright (C) 2020, Society of Photo-Optical Instrumentation Engineers.

Copying of material in this book for internal or personal use, or for the internal or personal use of specific clients, beyond the fair use provisions granted by the U.S. Copyright Law is authorized by SPIE subject to payment of copying fees. The Transactional Reporting Service base fee for this volume is $\$ 18.00$ per article (or portion thereof), which should be paid directly to the Copyright Clearance Center (CCC), 222 Rosewood Drive, Danvers, MA 01923. Payment may also be made electronically through CCC Online at copyright.com. Other copying for republication, resale, advertising or promotion, or any form of systematic or multiple reproduction of any material in this book is prohibited except with permission in writing from the publisher. The CCC fee code is 0277$786 \mathrm{X} / 20 / \$ 18.00$.

Printed in the United States of America.

Publication of record for individual papers is online in the SPIE Digital Library.

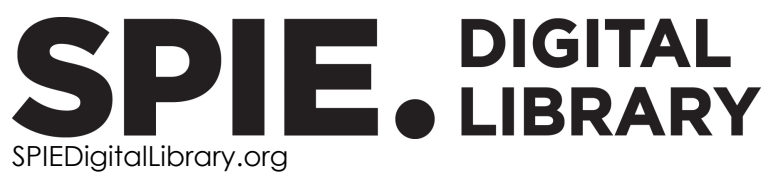

Paper Numbering: Proceedings of SPIE follow an e-First publication model. A unique citation identifier (CID) number is assigned to each article at the time of publication. Utilization of CIDs allows articles to be fully citable as soon as they are published online, and connects the same identifier to all online and print versions of the publication. SPIE uses a seven-digit CID article numbering system structured as follows:

- The first five digits correspond to the SPIE volume number.

- The last two digits indicate publication order within the volume using a Base 36 numbering

system employing both numerals and letters. These two-number sets start with 00, 01, 02, 03, 04,

05, 06, 07, 08, 09, 0A, OB ... 0Z, followed by 10-1Z, 20-2Z, etc. The CID Number appears on each

page of the manuscript. 


\title{
Contents
}

\author{
vii Authors \\ ix Conference Committee
}

TERAHERTZ AND SUB-TERAHERTZ DEVICES

1127903 Si, SiGe, InP, III-N, and p-diamond FETs and HBTs for sub-terahertz and terahertz applications (Invited Paper) [1 1279-2]

$1127904 \quad 1 T H z$ one-sided directional slot antenna on a chip connected with InAs HEMT [1 1279-3]

1127905 Sub-terahertz detection by fin-shaped GaN/AIGaN transistors [1 1279-4]

INFRARED DEVICES, TECHNOLOGY, AND APPLICATIONS

1127907 Performance enhancement of quantum dot infrared photodetector by periodic pillar array [11279-6]

1127908 Advanced broadband MEMS infrared emitter based on high-temperature-resistant nanostructured surfaces and packaging solutions for harsh environments [11279-7]

1127909 Semiconducting amorphous Y-Ba-Cu-O: an attractive material for fast and sensitive thermal sensing in the NIR to THz range [1 1279-8]

TERAHERTZ FRONTIERS

11279 OD Vectorial terahertz pulse time-domain holography for broadband optical wavefront sensing [11279-12]

THz IMAGING AND SAMPLING

11279 ol Spatial sampling of terahertz fields with subwavelength accuracy via probe beam encoding [11279-18]

TERAHERTZ LAYER THICKNESS EVALUATION

$112790 \mathrm{~J}$ Simulation of terahertz waves in multilayer coatings for non-contact thickness measurements of top layers [11279-19] 
$11279 \mathrm{OL} \quad$ Optical thickness of a plant leaf measured with THz pulse echoes [11279-21]

FAST-SCANNING TERAHERTZ TDS SYSTEMS

$1127900 \quad$ Fast thickness gauging with an ECOPS-based terahertz time-domain system (Invited Paper) [11279-24]

TERAHERTZ CROSS-CORRELATION SYSTEMS

11279 OR Stable THz wave generations using laser chaos (Invited Paper) [11279-27]

PHOTONIC TERAHERTZ SYSTEMS

$112790 X \quad$ Terahertz spectrometer based on widely tunable injection-seeded terahertz parametric generation and detection for identifying pharmaceutical materials [11279-33]

TERAHERTZ COMPONENTS

11279 OY Graphene-based highly efficient C-shaped metasurface for terahertz absorber [11279-34]

1127910 THz emission from semiconductors using excitation by a tilted pulse front [1 1279-36]

TERAHERTZ DEVICES

1127913 Broadband terahertz radiation produced in an AgGaSe2 crystal [11279-39]

1127914 Room-temperature broadband terahertz detector based on three-dimensional graphene [11279-40]

\section{TERAHERTZ IMAGING}

1127915 Terahertz imaging with nonlinear quantum-cascade-laser source [11279-41]

1127917 Rapid nondestructive evaluation of defects in GFRP composites using terahertz line scanner [11279-43]

1127918 Resolution enhancement of THz imaging based on Fourier-space spectrum detection [11279-44]

iv 


\section{CROSS CUTTING TECHNOLOGIES}

11279 1B The principle of measuring instantaneous frequent and dynamic linewidth of frequency swept lightwaves [11279-47]

11279 1C Simultaneous enhancement of dynamic range and sensitivity in slope-assisted Brillouin optical time-domain analyzers via gain spectrum engineering [1 1279-48]

\section{TERAHERTZ DEVELOPMENTS}

$112791 Q \quad$ Simulation study and experimental confirmation of a tunable terahertz negative-refractive index metamaterial [11279-62]

\section{NOVEL TECHNOLOGIES AND APPROACHES}

11279 IR Thin film lithium niobate optical modulators for THz frequency applications [11279-63]

11279 is Hydration studies of nanosilica incorporated cement using mid-infrared and terahertz spectroscopy [11279-64]

11279 IT Improving the linearity of silicon ring modulators by manipulating the quality factor and operation wavelength [11279-65]

$112791 \mathrm{U}$ Microscopic conductivity and ultrafast carrier dynamics in molybdenum-based MXenes: THz spectroscopy study [11279-66]

11279 IV Generation of kilovolt, picosecond electric pulses by coherent combining in optoelectronic system [1 1279-67]

\section{CHARACTERIZATION AND APPLICATIONS}

11279 IW Investigation on the excess noise in Brillouin optical time domain analysis due to stimulated Brillovin scattering [11279-68]

$112791 \mathrm{X}$ Degradation effects and origin in H-terminated diamond MESFETs [1 1279-69]

11279 1Y Investigation of single-mode anti-resonant hollow-core THz fibers [11279-70]

\section{POSTER SESSION}

1127921 Complex-domain sparse imaging in terahertz pulse time-domain holography with balance detection [11279-16] 
1127922 The nonlinear tuning technique of a DFB laser using frequency predistortion procedures [1 1279-72]

1127924 Tunable linear-step-swept light source based on time-delayed spectrum stitching technique [11279-74]

1127926 Modeling of plasmonic semiconductor THz antennas in square and hexagonal array arrangements [11279-76]

11279 2C High-resolution motorized $x-z$ translation stage to scan 3D objects using terahertz technology [11279-82]

$112792 \mathrm{D}$ Characterization of silicon in the terahertz [11279-83]

$112792 \mathrm{E} \quad$ Sub-pixel motion estimation for terahertz imaging [1 1279-84] 


\section{Authors}

Numbers in the index correspond to the last two digits of the seven-digit citation identifier (CID) article numbering system used in Proceedings of SPIE. The first five digits reflect the volume number. Base 36 numbering is employed for the last two digits and indicates the order of articles within the volume. Numbers start with 00, 01, 02, 03, 04, 05, 06, 07, 08, 09, OA, OB...0Z, followed by 10-12, 20-2Z, etc.

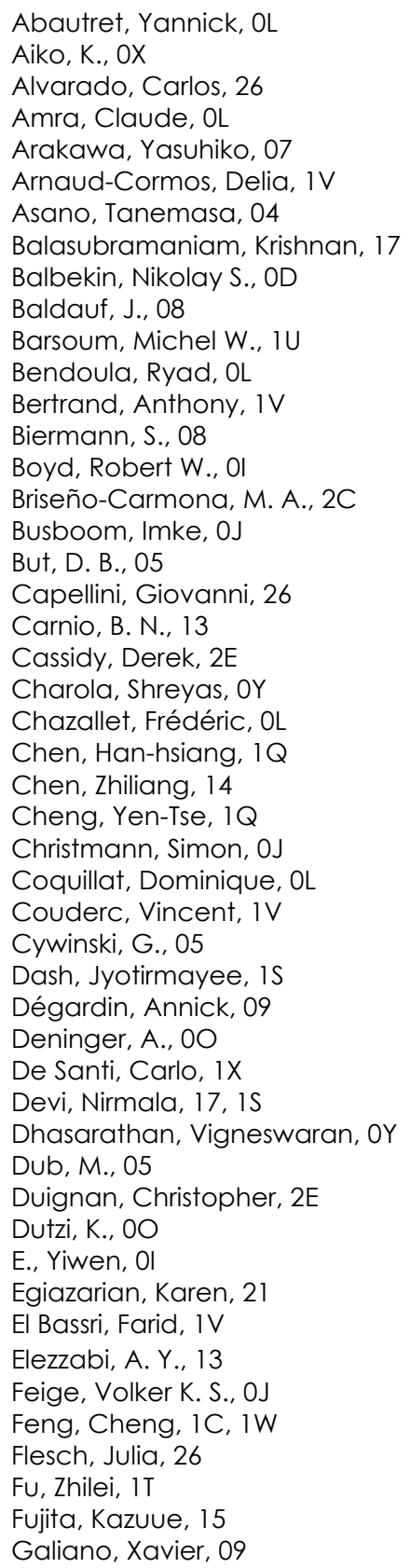

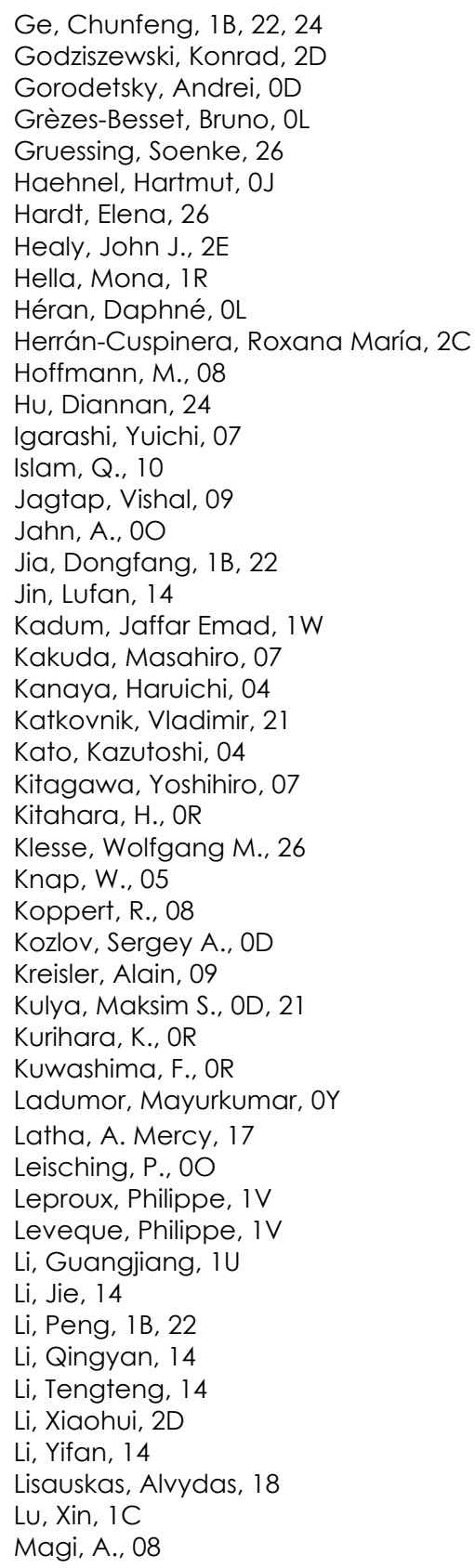


Meneghesso, G., $1 X$

Meneghini, M., $1 \mathrm{X}$

Meng, F., 10

Mohara, M., OX

Morikawa, O., OR

Müller, L., 08

Nakajima, M., OR

Nakanishi, Atsushi, 15

Nardo, A., 1X

Natu, Varun, $1 \mathrm{U}$

Olivares-Pérez, Arturo, 2C

Ono, T., OX

Ortlepp, T., 08

Pagnoux, Dominique, IV

Patel, Shobhit K., OY

Pavanello, L., $1 \mathrm{X}$

Paz-Martí-nez, Gaudencio, 2C

Pesala, Bala, 17, 1s

Petrov, Nikolay V., OD, 21

Piehler, Jacob, 26

Prystawko, P., 05

Puppe, T., 00

Qin, Jiaqi, 24

Rabiei, Payam, $1 \mathrm{R}$

Rajagopal, Prabhu, 17

Ray, Shaumik, is

Rayapureddy, Supriyanka, 17

Roemer, Friedhard, 26

Rollinson, John, $1 \mathrm{R}$

Roskos, Hartmut G., 10, 18

Rumyantsev, S., 05

Sachse, P., 08

Sai, P., 05

Sakowicz, M., 05

Sasmal, Saptarshi, is

Satoh, Tetsuro, 07

Satozono, Hiroshi, 15

Schmauss, B., 00

Schneider, Thomas, 1C, IW

Schunemann, P. G., 13

Sheng, Quan, $1 Y$

Sheridan, John T., 18, 2D, 2E

Shi, Teng, $1 \mathrm{U}$

Shi, Wei, 1Y

Shibuya, Akinobu, 07

Shibuya, Taizo, 07

Shimura, K., OX

Shirane, Masayuki, 07

Shirao, T., OR

Shur, Michael, 03

Soriano, Gabriel, OL

Sun, Da-Wen, 2D

Sun, Shuai, $1 Y$

Takigawa, Ryo, 04

Tanaka, Tomo, 07

Tani, M., OR

Tasaki, Kohei, 04

Tibken, Bernd, 0J

Titova, Lyubov V., $1 \mathrm{U}$

Toroghi, Seyfollah, IR
Treviño-Palacios, Carlos G., 2C

Tsui, Chi-leung, 1Q

Vallejo-Mendoza, Rosaura, 2C

Verona Rinati, G., IX

Verona, C., IX

Vieweg, N., 00

Villa-Hernández, Joan Manuel, 2C

Wan, Min, 18, 2D, 2E

Wang, Wei-Chih, 1Q

Wang, Xiaofei, IT

Wang, Zhaoying, 1B, 22, 24

Wedrich, K., 08

Wehbi, Sahar, $1 \mathrm{~V}$

Wen, Lai, 24

Wilke, Ingrid, $1 \mathrm{R}$

Williams, Kaia, Ol

Witzigmann, Bernd, 26

Xia, Penghui, $1 T$

Yahyapour, M., 00

Yamamoto, K., OR

Yan, Zhongbao, $1 Y$

Yang, Jiewei, 1B, 22

Yang, Tianxin, 1B, 22, 24

Yao, Jianquan, 14, 1 Y

Yashchyshyn, Yevhen, 2D

You, Changjiang, 26

Yu, Hui, $1 T$

Yu, Yu, 14

Yuan, Hui, 10, 18

Yuan, Quan, 24

Zanoni, E., $1 \mathrm{X}$

Zawilski, K. T., 13

Zerrad, Myriam, OL

Zhang, Guo, $1 Y$

Zhang, Qiang, IT

Zhang, X.-C., Ol

Zhang, Yao, $1 Y$

Zhang, Yating, 14

Zhao, Hongliang, 14

Zhao, Jiapeng, $\mathrm{Ol}$ 


\section{Conference Committee}

Symposium Chairs

Sailing He, KTH Royal Institute of Technology (Sweden) and Zhejiang University (China)

Yasuhiro Koike, Keio University (Japan)

Symposium Co-chairs

Connie J. Chang-Hasnain, University of California, Berkeley (United States)

Graham T. Reed, Optoelectronics Research Center, University of Southampton (United Kingdom)

Program Track Chairs

James G. Grote, Photonics Engineering Consultant (United States)

Shibin Jiang, AdValve Photonics, Inc. (United States)

\section{Conference Chairs}

Laurence P. Sadwick, InnoSys, Inc. (United States)

Tianxin Yang, Tianjin University (China)

\section{Conference Program Committee}

René Beigang, Technische Universität Kaiserslautern (Germany)

Jianji Dong, Huazhong University of Science and Technology (China)

Frank Ellrich, Technische Hochschule Bingen (Germany)

Fabian Friederich, Fraunhofer-Institut für Techno- und

Wirtschaftsmathematik ITWM (Germany)

Robert H. Giles, University of Massachusetts Lowell (United States)

R. Jennifer Hwu, InnoSys, Inc. (United States)

Mona Jarrahi, University of California, Los Angeles (United States)

Daniel Molter, Fraunhofer-Institut für Techno- und

Wirtschaftsmathematik ITWM (Germany)

J. Anthony Murphy, National University of Ireland, Maynooth (Ireland)

Créidhe O'Sullivan, National University of Ireland, Maynooth (Ireland)

Kyung Hyun Park, Electronics and Telecommunications Research Institute (Korea, Republic of)

Alessia Portieri, TeraView Ltd. (United Kingdom)

Marco Rahm, Technische Universität Kaiserslautern (Germany)

Jinghua Teng, Institute of Materials Research and Engineering

(Singapore) 
Michael Weibel, Joint Research and Development, Inc. (United States)

Jiangfeng Zhou, University of South Florida (United States)

\section{Session Chairs}

1 Terahertz and Sub-Terahertz Devices

Laurence P. Sadwick, InnoSys, Inc. (United States)

Tianxin Yang, Tianjin University (China)

2 Infrared Devices, Technology, and Applications

Tianxin Yang, Tianjin University (China)

Laurence P. Sadwick, InnoSys, Inc. (United States)

3 Terahertz Frontiers

Laurence P. Sadwick, InnoSys, Inc. (United States)

R. Jennifer Hwu, InnoSys, Inc. (United States)

$4 \quad$ THz Imaging and Sampling

Robert H. Giles, University of Massachusetts Lowell (United States)

Kyung Hyun Park, Electronics and Telecommunications Research Institute (Korea, Republic of)

5 Terahertz Layer Thickness Evaluation

Fabian Friederich, Fraunhofer-Institut für Techno- und Wirtschaftsmathematik ITWM (Germany)

Daniel Molter, Fraunhofer-Institut für Techno- und Wirtschaftsmathematik ITWM (Germany)

6 Fast-Scanning Terahertz TDS Systems

Daniel Molter, Fraunhofer-Institut für Techno- und Wirtschaftsmathematik ITWM (Germany)

Fabian Friederich, Fraunhofer-Institut für Techno- und Wirtschaftsmathematik ITWM (Germany)

7 Terahertz Cross-Correlation Systems

Fabian Friederich, Fraunhofer-Institut für Techno- und Wirtschaftsmathematik ITWM (Germany)

Daniel Molter, Fraunhofer-Institut für Techno- und Wirtschaftsmathematik ITWM (Germany)

8 Photonic Terahertz Systems

Daniel Molter, Fraunhofer-Institut für Techno- und Wirtschaftsmathematik ITWM (Germany)

Fabian Friederich, Fraunhofer-Institut für Techno- und Wirtschaftsmathematik ITWM (Germany) 
9 Terahertz Components

Kyung Hyun Park, Electronics and Telecommunications Research Institute (Korea, Republic of)

Robert H. Giles, University of Massachusetts Lowell (United States)

10 Terahertz Devices

Daniel Molter, Fraunhofer-Institut für Techno- und Wirtschaftsmathematik ITWM (Germany)

Fabian Friederich, Fraunhofer-Institut für Techno- und Wirtschaftsmathematik ITWM (Germany)

11 Terahertz Imaging

Fabian Friederich, Fraunhofer-Institut für Techno- und Wirtschaftsmathematik ITWM (Germany)

Daniel Molter, Fraunhofer-Institut für Techno- und Wirtschaftsmathematik ITWM (Germany)

12 Cross Cutting Technologies

Laurence P. Sadwick, InnoSys, Inc. (United States)

R. Jennifer Hwu, InnoSys, Inc. (United States)

13 Fundamental Research in Terahertz Physics

Marco Rahm, Technische Universität Kaiserslautern (Germany)

Laurence P. Sadwick, InnoSys, Inc. (United States)

14 RF/Microwave/Millimeter-Wave

Laurence P. Sadwick, InnoSys, Inc. (United States)

Tianxin Yang, Tianjin University (China)

15 Terahertz Developments

Tianxin Yang, Tianjin University (China)

Laurence P. Sadwick, InnoSys, Inc. (United States)

16 Novel Technologies and Approaches

Robert H. Giles, University of Massachusetts Lowell (United States)

Kyung Hyun Park, Electronics and Telecommunications Research Institute (Korea, Republic of)

17 Characterization and Applications

Laurence P. Sadwick, InnoSys, Inc. (United States)

Tianxin Yang, Tianjin University (China) 
Proc. of SPIE Vol. 11279 1127901-12

Downloaded From: https://www.spiedigitallibrary.org/conference-proceedings-of-spie on 26 Apr 2023 Terms of Use: https://www.spiedigitallibrary.org/terms-of-use 